\title{
Clinical versus ultrasound examination in the evaluation of hepatosplenic schistosomiasis mansoni in endemic areas
}

\section{Carolina Coimbra Marinho, Izabela Voieta, Letícia Martins Azeredo**, Marina Pires Nishi, Thaís Sanai Batista, Ana Carolina Figueiredo Pereira, José Carlos Serufo, Leonardo Campos de Queiroz, Raiza Ruiz-Guevara*, Carlos Maurício Antunes, Aluízio Prata*, José Roberto Lambertucci/ ${ }^{+}$}

\begin{abstract}
Programa de Pós-Graduação em Ciências da Saúde: Infectologia e Medicina Tropical, Faculdade de Medicina, Universidade Federal de Minas Gerais, Departamento de Clínica Médica, Av. Alfredo Balena 190, s/3045, 30130-100 Belo Horizonte, MG, Brasil *Faculdade de Medicina do Triângulo Mineiro, Uberaba, MG, Brasil **Serviço de Radiologia do Hospital das Clínicas da Universidade Federal de Minas Gerais, Belo Horizonte, MG, Brasil
\end{abstract}

The best way to appraise the size of abdominal organs remains undefined. Herein we compare the size of liver and spleen in hepatosplenic schistosomiasis using clinical and ultrasound (US) examination, and the size of the organs measured by US with their visualization below the costal margin ("palpable by US"). For this study, 411 individuals from an endemic area for schistosomiasis mansoni in Brazil have been selected. We found that palpable spleens and left liver lobes are larger than non palpable ones. Also, $23 \%$ of normal spleens measured by US were palpable on clinical examination, and $22 \%$ of spleens increased in size on US were non palpable. A total of $21 \%$ of normal spleens were "palpable by US". We also found 54\% of normal sized right liver lobes palpable on clinical examination, whilst $54 \%$ of the increased livers, measured by US, were non palpable. About $76 \%$ of normal right liver lobes were "palpable by US". We conclude that the association of clinical, ultrasound and magnetic resonance imaging (MRI) examinations, in the near future, should give the investigators the necessary tools to perform a more accurate clinical diagnosis of hepatosplenic schistosomiasis mansoni.

Key words: schistosomiasis - ultrasound - physical examination - spleen - liver - portal hypertension

Ultrasound (US) has been routinely used in the evaluation of hepatosplenic schistosomiasis mansoni for the last 20 years (Pinto-Silva et al. 1994, Lambertucci et al. 1996, Gesparcher-Lara et al. 1997, Martins et al. 1998). The use of US is justified by its low cost, and portability which made it possible to be used in field-based studies. However, the best way to appraise the size of abdominal organs remains undefined.

More objective criteria to evaluate liver fibrosis and portal hipertension in schistosomiasis mansoni, using US, appeared in 1996 and were revised in 2000: the evaluation of portal and splenic vein diameters, the disclosure of collateral veins, the diagnosis of ascites, the measurement of the wall of portal branches, the sizes of liver and spleen, the identification of gallbladder wall thickening, and the image patterns of the liver (Richter et al. 2000, 2001).

It is worth mentioning that the palpation of liver and spleen below the right and left costal margins and xiphoid process is accepted by most physicians as a sign of liver and spleen enlargement.

In this study, the sizes of spleen, right and left liver lobes longitudinal diameters, measured by US, are compared to the finding of palpable organs during physical

${ }^{+}$Corresponding author: lamber@uai.com.br

Received 25 May 2006

Accepted 26 June 2006 examination, to verify whether a palpable organ is always increased, and to evaluate whether a palpable organ below the costal margin is also visualized by US as being below the costal margin ("palpable by US").

\section{PATIENTS AND METHODS}

Patients - The population of Brejo do Espírito Santo, a rural community of Santa Maria da Vitória in the state of Bahia, Brazil, was examined, every 4 years, since 1976 by one of us (AP). A total of 3766 people have been followed up. Prevalence of schistosomiasis, determined by parasitological stool examinations, using the Kato-Katz technique, had been identified to be $75 \%$ in 1976 . After the implementation of disease control measures, significant improvement has occurred, and prevalence of schistosomiasis dropped down to $1.8 \%$ by 2004 (Guevara 2005).

In October 2004 a convenience sample, including 411 individuals between 3 and 92 years of age were selected for this study. This is a transversal study and the individuals who agreed to participate were allocated in three groups, according to clinical evaluation: (1) 41 patients with current clinical data compatible with liver fibrosis (hepatosplenomegaly, and hardened, and/or nodular liver), 11 of which had previously undergone splenectomy; (2) 102 patients with previous history of liver fibrosis, but with no current clinical data of liver fibrosis, 17 of which had previously undergone splenectomy; (3) 268 individuals who have never presented clinical data compatible with liver fibrosis. The participants were submitted to clinical and US examinations and the collected information was stored in a data bank elaborated with appropriate 
software. Twenty nine individuals were excluded of the study: 5 individuals aged 5 years or less who did not collaborate with US examination, and 24 individuals whose sizes of liver and spleen were not measured or the organs visualization below the costal margin by US were not recorded in the research protocol. Therefore, 382 individuals $(92.9 \%)$ remained in the study.

Physical examination - Physical examination was conducted by two physicians, AP and JRL, in an independent fashion. After the examination, the two exams were compared and when any difference existed, the examiners searched for a consensus, in order to create only one register for each patient. Weight, height, vital signs, and cardio-respiratory evaluation were watched and registered. During abdominal examination ascites and collateral circulation were searched. Abdominal palpation was performed with patients in the dorsal decubitus, during deep breath. Liver and spleen have been considered to be palpable when liver and spleen borders were felt below the costal margins by both examiners. The greatest distance between liver and spleen borders and respective costal margins was also independently measured by both examiners.

US - Sonographic examination was conducted by a specialist trained in the application of the Niamey's protocol, 1996, revised in 2000 (Richter et al. 2000, 2001). A portable GE Logic Book equipment was used with a 2.5 to $5 \mathrm{MHz}$ polifrequencial convex transducer witch allows storage of raw data in Dicom format (specific medical images protocol, without loss of quality) for future re-evaluation.

To verify whether the palpable organ by physical examination was also visualized by US below the costal margin, the transducer was positioned across the right costal margin for right liver lobe observation and across left costal margin for spleen observation. The organ move- ment was thus observed through the respiratory cycle. In case the organ continued to be visualized below the costal margin, at the end of inhalation, it was said to be "palpable by US" (Fig. 1). The manoeuvre was not applied to the left liver lobe since in most cases it is already visualized below the xiphoid process during clinical examination of healthy individuals.

Ethics - This work was approved by the Ethic Board of Universidade Federal de Minas Gerais and by the Brazilian Board of Ethics in Medical Research. All participants have given written authorization at the time of inclusion in the study.

Viral hepatitis - Prevalence of hepatitis B and C was determined in the study population. The prevalence of hepatitis B was $17 \%$, when HBsAg and anti-HBc were included (HBs and anti-HBc EIA, Medical Biological Service, Milano, Italy), and $0.7 \%$ for $\mathrm{HBsAg}$ alone (chronic carriers). Prevalence of hepatitis $\mathrm{C}$ was $0.7 \%$, using an ELISA anti-HCV (Detect-HCV 3.0, Adaltis, Montreal, Canada).

Statistical analysis - Data analysis was performed using the program EPI-Info, version 3.3.2/2005. Student's $t$ test and Kruskal-Wallis were used to compare means, and McNemar's test was used to analyze the differences between proportions. The concordance between diagnostic tests was defined by kappa analysis.

\section{RESULTS}

Spleen-The mean longitudinal diameter of the spleen measured by US was $75.9 \mathrm{~mm}$ for non palpable spleen and $107.6 \mathrm{~mm}$ for palpable spleen on physical examination $(\mathrm{p}=$ 0.00 ). In the group "palpable by US", mean longitudinal diameter was $73.7 \mathrm{~mm}$ for non palpable organs and $115 \mathrm{~mm}$ for palpable ones $(p=0.00)$.

Fig. 2 shows the distribution of patients with palpable and non palpable spleen by physical and US examination,

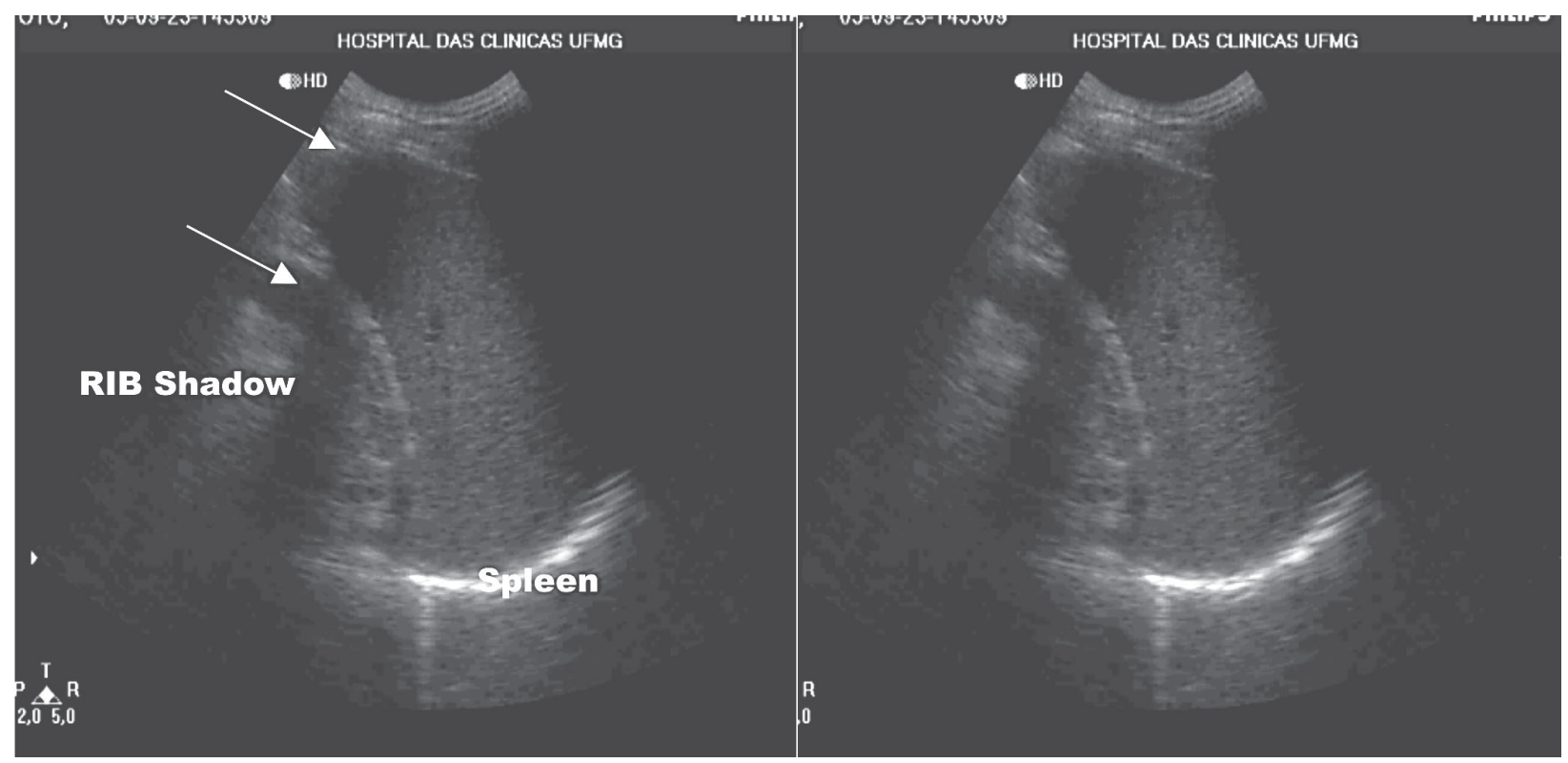

Fig. 1: image of the spleen visualized below the costal margin (white arrows point the shadow of the rib). 


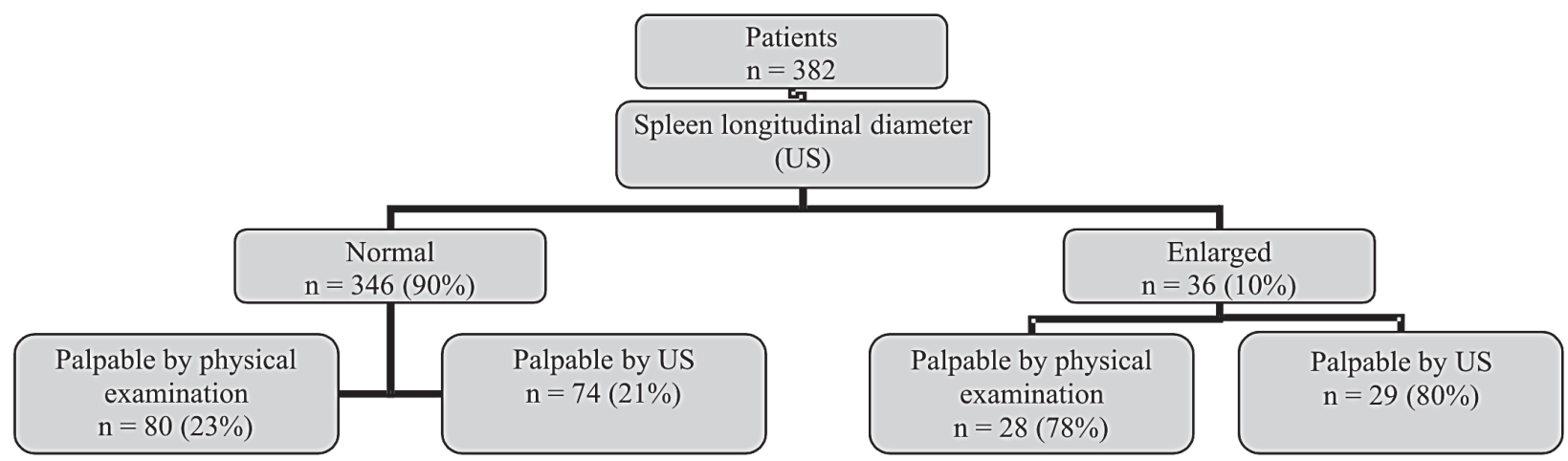

Fig. 2: distribution of patients according to spleen longitudinal diameter measured by ultrasound.

according to US measure of longitudinal diameter. Spleen longitudinal diameters were normal in 346 patients $(90 \%)$. Among them, 80 (23\%) had a palpable spleen by clinical examination and $74(21 \%)$ had the spleen "palpable by US". Twenty eight out of 36 patients $(78 \%)$ who have had an increased longitudinal spleen diameter by US had a palpable spleen in physical examination, and $29(80 \%)$ had the organ "palpable by US".

In Table I the number of patients with "palpable and non palpable spleen by US" is compared to those palpable and non palpable by physical examination. Both methods showed similar results when evaluating spleen enlargement, although discordance existed in 67 cases $(17 \%)$.

Right liver lobe - Mean longitudinal diameter of right liver lobe measured by US was $113.2 \mathrm{~mm}$ for non palpable and $111.8 \mathrm{~mm}$ for palpable right liver lobe on physical examination $(\mathrm{p}=0.51)$. In the group "palpable by US", mean longitudinal diameter was $105.2 \mathrm{~mm}$ for non palpable and $114.6 \mathrm{~mm}$ for palpable right liver lobe $(\mathrm{p}=0.0001)$.

In Fig. 3 is depicted the distribution of patients with palpable and non palpable right liver lobe by physical and US examination, according to US measure of longitudinal diameters. Right liver lobe longitudinal diameters were normal in 360 patients (94\%). Among them, 195 (54\%) had a palpable liver by clinical examination and $274(76 \%)$ had the organ "palpable by US". Ten out of 22 patients $(46 \%)$ who have had an increased longitudinal right liver

\section{TABLE I}

Spleen palpation by ultrasound (US) versus clinical palpation of the spleen

\begin{tabular}{lccc}
\hline \multirow{2}{*}{$\begin{array}{l}\text { Clinical palpation } \\
\text { of the spleen }\end{array}$} & $\begin{array}{c}\text { Non } \\
\text { palpable (\%) }\end{array}$ & Palpable (\%) & Total (\%) \\
\cline { 2 - 4 } $\begin{array}{l}\text { Non palpable } \\
\text { Palpable }\end{array}$ & $243(87)$ & $31(30)$ & $274(72)$ \\
Total & $36(13)$ & $72(70)$ & $108(28)$ \\
\hline
\end{tabular}

kappa $=0.5614$

lobe diameter by US had a palpable right liver lobe in physical examination, and 21 (95\%) had the organ "palpable by US".

In Table II the number of patients with "palpable and non palpable right liver lobe by US" is compared to those palpable and non palpable by physical examination. The methods showed no agreement in the evaluation of the right liver lobe enlargement. Results diverged in 160 cases $(42 \%)$.

Left liver lobe - The mean longitudinal diameters of left liver lobe measured by US was $79.6 \mathrm{~mm}$ for non palpable and $81.3 \mathrm{~mm}$ for palpable left liver lobes on physical examination $(\mathrm{p}=0.32)$.

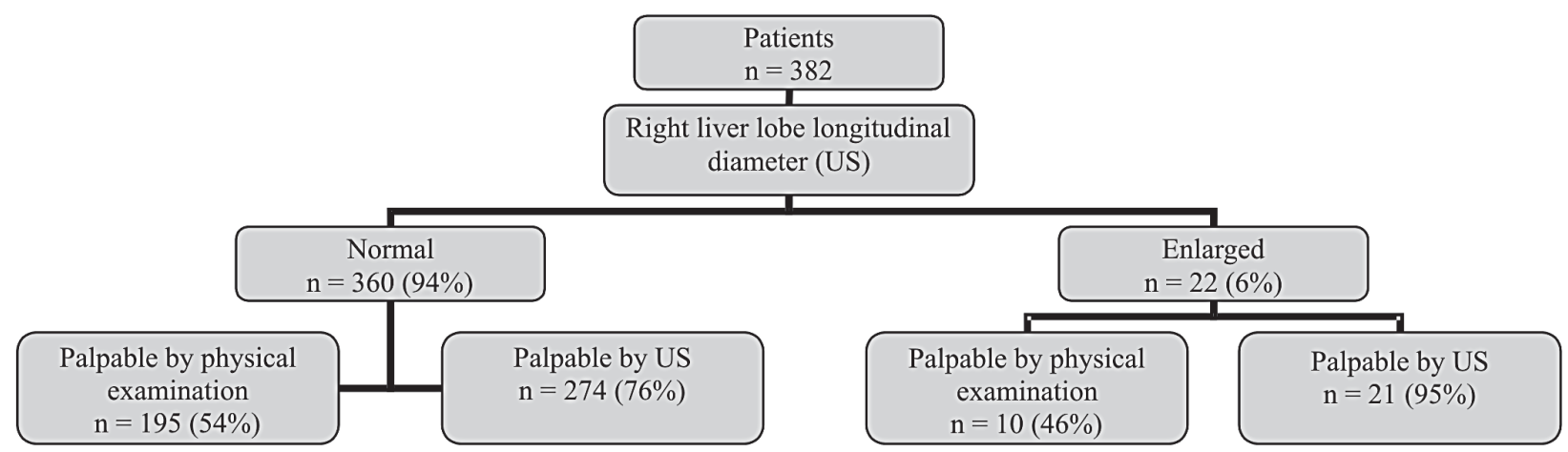

Fig. 3: distribution of patients according to right liver lobe longitudinal diameters measured by ultrasound. 
A total of 227 patients (59\%) presented a normal left liver lobe size. Among those, 114 (50\%) considered normal had the liver lobe palpable by physical examination. One hundred fifty five patients $(41 \%)$ had an enlarged left liver lobe when measured by US, and 64 out of $155(41 \%)$ had a non palpable organ below the xiphoid process $(\mathrm{p}=$ 0.126 ).

Comparison between clinical and sonographic liver examination - One single patient presented nodular liver surface by physical examination $(0.5 \%)$ and six patients presented nodular liver margin by US examination (3\%). Ninety seven patients $(48 \%)$ presented a hardened liver on physical examination, with fibrosis present on US in 72 (35\%). The liver was palpable in 205 cases, however, no concordance was found between consistency of the liver and the presence of fibrosis in US in 70 cases (34\%).

\section{TABLE II}

Right liver lobe palpation by ultrasound (US) versus physical examination

US

\begin{tabular}{lccc} 
Right liver & & & \\
\cline { 2 - 4 } lobe palpation & $\begin{array}{c}\text { Non } \\
\text { palpable }(\%)\end{array}$ & Palpable (\%) & Total (\%) \\
\hline $\begin{array}{l}\text { Non palpable } \\
\text { Palpable }\end{array}$ & $52(60)$ & $125(42)$ & $177(46)$ \\
\hline Total & $35(40)$ & $170(58)$ & $205(54)$ \\
\hline
\end{tabular}

kappa $=0.1275$

TABLE III

Fibrosis by ultrasound (US) versus liver consistency by physical examination

\begin{tabular}{lccc}
\hline Physical examination & \multicolumn{3}{c}{ Fibrosis on US } \\
\cline { 2 - 4 } Liver consistency & Absent (\%) & Present (\%) & Total \\
\hline Normal & $86(64)$ & $22(30)$ & $108(53)$ \\
Hardened & $48(36)$ & $49(70)$ & $97(47)$ \\
\hline Total & $134(100)$ & $72(100)$ & $205(100)$ \\
\hline
\end{tabular}

kappa $=0.3056$

\section{DISCUSSION}

Twenty three percent of normal size spleens as measured by US were palpable on clinical examination. Also, $20 \%$ of the spleens considered to be enlarged by US were non palpable during physical examination. As to right liver lobe, more than half of palpable livers were of normal size on US. For spleens and livers "palpable by US" similar results were obtained. Clinical evaluation of liver consistency had only weak correlation with the presence of fibrosis on US examination.

Gesparcher-Lara et al. (1998), studying the value of spleen palpation in the evaluation of morbidity in schistosomiasis mansoni, found low sensitivity and positive predictive values for a palpable spleen to diagnose hepatosplenic schistosomiasis in endemic areas. Lambertucci et al. $(2000,2001)$ and Cota et al. (2006), in field- based studies, identified four groups of patients with hepatosplenic schistosomiasis and proposed a classification which was based on clinical and US examinations. The data of the present study obtained in an endemic area of Bahia confirms previous findings. Isolated physical examination underestimates enlarged spleens which are non palpable and overestimates palpable spleens which are not enlarged when measured by US. In addition, our findings do not support the classical semiologic statement that a palpable spleen is always enlarged.

Right liver lobes identified below the costal margin both by physical examination and "US palpation" proved to be of normal sizes in most cases.

In this study we have used US as the gold standard to measure the size of liver and spleen. This decision may have led us to make mistakes. What is the precision of US to measure the size of the organs (liver and spleen, for example)? We do not know the answer. Let us point out one limitation of US for this purpose. For instance, the upper borders of the liver and spleen are darkened by the air present in the lower portions of the lungs, and therefore, the limits between liver and spleen and the echogenic line of the lungs images are arbitrarily considered (extrapolated) to be the reference to measure the longitudinal diameters of the organs (Niederau et al. 1983).

The "US palpable" technique was used for the first time in this work. Our intention was to see and record, using US resources, the spleen or liver below the costal margin, instead of perceiving it by palpation. Possible advantages were: to avoid the inconvenience of thickened abdominal walls (either by obesity or well developed abdominal muscles) and of huge ascites. In fact, the manoeuvre allows a qualitatively evaluation of the organ below the costal margin during respiratory movements similar to manual palpation. Easily and quickly performed, it eliminates errors inherent to the way the organs are measured by US, discussed in the previous paragraph, and apparently decreases the inter-examiner error due to the subjectivity of abdominal palpation.

Left liver lobe palpation below the xyphoid process did not correlate in this study with organ enlargement. Another study from a low transmition area of schistosomiasis in Venezuela failed to find any significant difference between the size of left liver lobe in S. mansoni infected and non infected individuals (Ruiz 2002). The shortcoming is that this study has not taken as reference the values for liver and spleen size proposed in the Niamey's protocol (Richter et al. 2000).

In a study from India, liver size estimated by US, was compared to physical examination. The authors found a very low precision of physical examination in identifying hepatomegaly (Joshi et al. 2004). This finding is in agreement with our study. However, in their work they used as reference values the sizes of the organs described in 1983 for a Caucasian population, without stratification for height (Niederau et al. 1983).

The reference values for organ sizes in the Niamey's protocol have been established, on its turn, based on a study performed in the population of a non endemic area for schistosomiasis in Senegal (Yazdanpanah et al. 1997). This emphasizes the importance of height and ethnic-de- 
pendent reference values to enhance the precision of US evaluation of abdominal organs. The extrapolation of reference normal values obtained from a sample of the Senegalese people to study a Brazilian population does not seem to be adequate. Hence, studies to establish normal US values for liver and spleen sizes in Brazil should be stimulated.

Still, clinical evaluation of liver consistency had only weak correlation with the presence of fibrosis on US examination. This observation reinforces the need to associate US and clinical examination to improve the quality of clinical evaluation.

More recently, the finding of periportal fibrosis in sonographic examination has been questioned when the authors used magnetic resonance imaging (MRI) to confirm the diagnosis of liver periportal fibrosis (Silva et al. 2006). A significant number of patients diagnosed as having periportal fibrosis by US where shown to have, by MRI, fat infiltration of the periportal tracts. For now, what appears to be the wisest approach is to use the information obtained by clinical, US and MRI examinations (whenever possible), to perform a more accurate clinical diagnosis of hepatosplenic schistosomiasis mansoni.

\section{REFERENCES}

Cota GF, Pinto-Silva RA, Antunes CMF, Lambertucci JR 2006. Ultrasound and clinical investigation of hepatosplenic schistosomiasis: evaluation of splenomegaly and liver fibrosis four years after mass chemotherapy with oxamniquine. $\mathrm{Am}$ J Trop Med Hyg 74: 103-107.

Gesparcher-Lara R, Pinto-Silva RA, Rayes AA, Drummond SC, Lambertucci JR 1997. Ultrasonography of periportal fibrosis in schistosomiasis mansoni in Brazil. Trans $R$ Soc Trop Med Hyg 91: 307-309.

Gesparcher-Lara R, Pinto-Silva RA, Serufo JC, Rayes AAM, Drummond SC, Lambertucci JR 1998. Splenic palpation for the evaluation of morbidity due to schistosomiasis mansoni. Mem Inst Oswaldo Cruz 93 (Suppl.): 245-248.

Guevara RJR 2005. Esquistossomose na Área Hiperendêmica de Brejo do Espírito Santo, com Seguimento de 29 Anos, $\mathrm{PhD}$ Thesis, Universidade Federal do Triângulo Mineiro, Uberaba.

Joshi R, Singh A, Janjoo N, Pai M, Kalantri SP 2004. Accuracy and reliability of palpation and percution for detecting hepatomegaly: a rural hospital-based study. Indian $J$ Gastroenterol 23: 171-174.

Lambertucci JR, Cota GF, Pinto-Silva RA, Serufo JC, Gesparcher-Lara R, Drummond SC, Antunes CM, Nobre V, Rayes AA 2001. Hepatosplenic schistosomiasis in fieldbased studies: a combined clinical and sonographic definition. Mem Inst Oswaldo Cruz 96 (Suppl.): 147-150.
Lambertucci JR, Gesparcher-Lara R, Pinto-Silva RA, Barbosa MM, Teixeira R, Barbosa HF, Serufo JC, Rezende DF, Drummond SC, Rayes AA 1996. The Queixadinha Project: morbidity and control of schistosomiasis in an endemic area in the northeast of Minas Gerais, Brazil. Rev Soc Bras Med Trop 29: 127-135.

Lambertucci JR, Serufo JC, Gesparcher-Lara R, Rayes AAM, Teixeira R, Nobre V, Antunes CMF 2000. Schistosoma mansoni: assessment of morbidity before and after control. Acta Trop 77: 101-109.

Martins MJ, Pinto-Silva RA, Serufo JC, Rayes AAM, Damasceno MPS, Martins MLV, Santos APS, Drummond SC, Bezerra MAS, Lambertucci JR 1998. Morbidity of schistosomiasis in an endemic area of northeast of the state of Minas Gerais in Brazil: a clinical and sonographic study. Mem Inst Oswaldo Cruz 93 (Suppl. 1): 243-244.

Niederau C, Sonnenberg A, Müller JE, Erckenbrecht JF, Scholten T, Fritsch WP 1983. Sonographic measurements of the normal liver, spleen, pancreas and portal vein. Radiology 149: 537-540.

Pinto-Silva RA, Abrantes WL, Antunes, CM, Lambertucci, JR 1994. Sonographic features of portal hypertension in schistosomiasis mansoni. Rev Inst Med Trop São Paulo 36: 355361 .

Richter J, Domingues ALC, Barata CH, Prata AR, Lambertucci JR 2001. Report of the second satellite symposium on ultrasound in schistosomiasis. Mem Inst Oswaldo Cruz 96 (Suppl.): 151-156.

Richter J, Hatz C, Campagne G, Bergquist NR, Jenkins JM 2000. Editors. Ultrasound in schistosomiasis. A practical guide to the standardized use of ultrasonography for the assessment of schistosomiasis related morbidity. World Health Organization, Geneva, Switzerland. TDR/STR/SCH/ 00.1

Ruiz R, Garassini M, Tombazzi C, Certad G, Bruces AC, Noya O, Noya BA 2002. Schistosomiasis mansoni in low transmition areas. Abdominal ultrasound. Mem Inst Oswaldo Cruz 97 (Suppl.): 153-159.

Silva LCS, Pereira ACF, Queiroz LC, Antunes CMF, Lambertucci JR 2006. Disagreement between ultrasound and magnetic resonance imaging in the identification of schistosomal periportal fibrosis. Mem Inst Oswaldo Cruz 101 (Suppl. I): 279-282.

Yazdanpanah Y, Thomas AK, Kardoff R, Talla I, Sow S, Niang M, Stelma FF, Decam C, Rogerie F, Gryseels B, Capron A, Doehring E 1997. Organometric investigations of the spleen and liver by ultrasound in Schistosoma mansoni endemic and nonendemic villages in Senegal. Am J Trop Med Hyg 57: 245-249. 
